\title{
De la malléabilité des structures syntaxiques dans l'interaction orale : le cas des constructions clivées
}

\author{
Elwys De Stefani \\ Université de Neuchâtel / Université de Berne \\ elwys.destefani@rom.unibe.ch
}

\section{Introduction}

Les recherches sur les constructions clivées ont été conduites, pour le français, surtout sur la base de documents écrits (dans une perspective diachronique : Muller, 2003 ; Wehr, 2005 ; Rouquier, 2007) ou sur des exemples tirés de l'oral, mais analysés de façon décontextualisée (cf. Vikner, 1973 ; Lambrecht, 2001 ; Blanche-Benveniste, 2001b). Cet article se propose d'étudier les constructions clivées telles qu'elles sont utilisées dans les échanges conversationnels. Le corpus que nous soumettrons à examen comprend environ vingt heures d'entretiens qui se sont déroulés entre un enquêteur et des groupes de quatre à six personnes, discutant de leurs expériences avec le bilinguisme. Le cadre théorique et méthodologique est celui de l'analyse conversationnelle (Sacks, 1992) et, plus particulièrement, de la linguistique interactionnelle (Ochs, Schegloff \& Thompson, 1996; Mondada, 2001) qui étudie les liens complexes qui existent entre les propriétés et possibilités du système linguistique d'une part et l'organisation dynamique de l'interaction sociale de l'autre. Cette approche - qui s'appuie essentiellement sur des données empiriques, récoltées en suivant les principes ethnographiques - examine les formes grammaticales au sein des pratiques langagières et des interactions sociales dans lesquelles les participants s'engagent. De ce point de vue, nous analyserons les constructions clivées non pas (uniquement) d'un point de vue fonctionnel, mais nous essayerons également de décrire les tâches interactionnelles et discursives que les locuteurs accomplissent lorsqu'ils utilisent un format clivé. Ceci permettra d'une part de repenser les définitions formelles et fonctionnelles avancées jusqu'ici, d'autre part nous pourrons esquisser une conception actionnelle de la grammaire, que nous considérons comme une ressource dont les acteurs sociaux se servent pour organiser et accomplir des activités sociales (et non pas uniquement pour produire des énoncés " acceptables ").

\subsection{La phrase clivée dans la tradition linguistique}

La phrase clivée a été isolée comme un objet de recherche au début du XX siècle, à travers les travauX qu'Otto Jespersen a entrepris sur la langue anglaise (cf. Jespersen, 1927, 1937). La dénomination avancée par le linguiste danois (cleft sentence) renvoie à la manière dont celui-ci a analysé les constructions clivées : il y reconnaissait, en effet, l'extraposition d'un élément constitutif d'une phrase canonique du type Sujet-Verbe-Objet résultant dans une structure communément décrite comme c'est X qui/que Y. De nombreux chercheurs maintiennent cette idée, entre autres Blanche-Benveniste (2001a, 2001b), qui reconnaît dans la clivage un dispositif d'extraction permettant de diviser la rection verbale en deux parties. ${ }^{1}$ Jespersen - qui a illustré ses réflexions par des exemples de phrases anglaises, françaises et danoises - a également avancé des remarques concernant la prosodie et l'effet pragmatique des clivées : ainsi, celles-ci se caractériseraient par une absence de pauses et par une intonation " cohérente " (Jespersen, 1937 : 84). En outre, l'élément initial it is (c'est) serait utilisé « as a demonstrative gesture to point at one particular part of the sentence to which the attention of the hearer is to be drawn especially » (ibid.: 86). L'extrait suivant illustre une construction que la plupart des linguistes n'hésiteraient probablement pas à décrire comme une construction clivée :

(1) corpus A, 249

1 B c'est elles qui s- ont développé des compétences

L'énoncé est initié par l'élément «c'est» suivi d'un syntagme nominal (le pronom « elles ») qui est décrit d'habitude comme étant le focus de la phrase. Le pronom relatif suivant («qui ») introduit une 
subordonnée («ont développé des compétences »), réalisée dans le cadre d'une autoréparation. Cet exemple s'accorde vraisemblablement avec une très grande partie des définitions qui ont été avancées pour les phrases clivées. Ainsi, pour Muller $(2003$ : 101) « les clivées sont des constructions comportant une proposition principale par le démonstratif $c e, c^{\prime}$, avec le verbe être suivi d'un groupe nominal et une subordonnée introduite par que ou un relatif, comportant elle-même un verbe conjugué ». Si à première vue cette définition semble être consensuelle, les analyses que nous mènerons dans la section 2 révéleront qu'elle exclut des occurrences bien fréquentes qui, selon nous, ne devraient pas être écartées de la discussion autour du format clivé. La définition de Muller (2003) est en effet restrictive par rapport au statut grammatical de l'élément qui suit la forme verbale c'est: si l'auteur prévoit l'occurrence d'un «groupe nominal » dans cette position syntaxique, l'analyse de données interactionnelles montrera que d'autres éléments grammaticaux (par exemple des syntagmes prépositionnels ou des adverbes) peuvent occuper cette place. La difficulté de la délimitation des constructions clivées d'autres formats semblables représente, de manière générale, un problème épineux de l'analyse syntaxique : dès lors, les définitions avancées dans une perspective fonctionnelle ont plutôt mis l'accent sur les caractéristiques informationnelles, pragmatiques des constructions clivées : en effet, un bref survol sur les définitions proposés à ce jour nous permet de constater que la plupart du temps elles concernent deux niveaux différents de l'analyse linguistique, à savoir les niveaux a) syntaxique et b) pragmatique $:^{2}$

a) La description originale des constructions clivées - comme résultant de la réorganisation syntaxique d'une phrase canonique présumée (Jespersen, 1927 ; 1937) - a marqué profondément les études suivantes, qui s'inscrivent généralement dans cette vision " transformationnelle ". Il ne paraît pas surprenant, par exemple, que les grammairiens générativistes se soient appropriés cette description des phrases clivées (cf. Lees, 1963 qui a proposé la première étude générativiste sur la phrase clivée en anglais). L'approche générativiste a également développé la notion de pseudo-clivée (Peters \& Bach 1968) pour décrire des phrases qui, selon les adeptes de Chomsky, représentent une étape intermédiaire lors de la transformation d'une phrase du type Sujet-Verbe-Objet en une phrase clivée (cf. Akmajian 1970). ${ }^{3}$ De ce fait, les phrases clivées sont souvent analysées ensemble avec les phrases pseudo-clivées (cf. Prince : 1978 ; Sornicola : 1988 ; Collins : 2006) et avec d'autres formats clivés - identifiés surtout dans la langue anglaise - comme les th-clefts (Ball : 1977) et les reversed wh-clefts ou pseudo-clivées renversées (Lambrecht : 2001). Ces constructions ont été décrites avant tout comme des structures syntaxiques " équivalentes " au niveau sémantique, dans le sens qu'elles renverraient toutes à une phrase canonique présumée.

b) D'un point de vue pragmatique, les constructions clivées sont habituellement décrites comme des dispositifs syntaxiques qui permettent de marquer le focus de la phrase (cf. Lambrecht, 2001 ; Wehr, 2005). Dans cette perspective, le focus est situé immédiatement après l'élément c'est, alors que la phrase relative suivante exprime une présupposition (Lambrecht, 2001 ; Katz, 2000). Dès lors, la construction clivée est perçue comme un dispositif syntaxique qui sert à placer le focus de la phrase en position initiale, permettant ainsi au locuteur a) de marquer une information nouvelle ou difficilement récupérable du contexte discursif et b) d'établir un contraste (cf. Jespersen, 1949: 147 ; Geluykens, 1988: 826). Rouquier (2007:172) explique l'effet contrastif en observant que l'unité suivant le c'est est généralement traitée comme faisant partie d'un ensemble d'éléments appartenant à un même paradigme : ainsi, dans la phrase c'est Athon que je vois venir, l'élément Athon ferait partie, selon l'auteure, d'un paradigme réunissant tous les objets que le locuteur pourrait voir venir. Bien que ces effets pragmatiques soient aussi amplement attestés dans notre corpus, il subsiste de nombreux cas qui ne peuvent pas être décrits de manière adéquate en recourant uniquement à une analyse de la structure informationnelle des énoncés. Comme nous le verrons dans la partie analytique de cet article, les constructions clivées contribuent également à la structuration du discours à plus large échelle, dans le sens qu'elles interviennent dans l'organisation séquentielle des activités.

L'approche que nous avançons ici se différencie des études précédentes puisqu'elle ne part pas du principe que les constructions clivées seraient " dérivées " de formats canoniques préexistants. Comme l'a souligné aussi Davidse (2000) en s'intéressant aux aspects sémantiques et pragmatiques des constructions clivées, la vision transformationnelle ne permet pas de décrire de manière adéquate le fonctionnement de ces formats syntaxiques. Dès lors, lorsque nous parlons de constructions clivées, nous pourrions être accusés 
d'incohérence, étant donné que le terme même renvoie implicitement à un processus de transformation syntaxique. Nous maintenons néanmoins ce terme, par convention, sans pour autant adhérer à la conception transformationnelle des formats clivés. Ceux-ci peuvent en effet êtres analysés en tant que phénomènes syntaxiques empiriquement attestés, sans présumer l'existence de phrases canoniques desquels ils seraient dérivés.

\subsection{Un problème de délimitation}

L'une des préoccupations de nombreux chercheurs tourne autour de la différentiation des structures clivées d'autres constructions qui leur ressemblent formellement, comme certaines relatives. Lorsque la deuxième partie de la phrase sert à spécifier un aspect de l'élément situé entre c'est et qui/que, on aurait affaire à une clause relative " traditionnelle". Dans cette perspective, l'exemple suivant serait analysé comme une relative et non pas comme une clivée :

(2) corpus D, 1877-1879

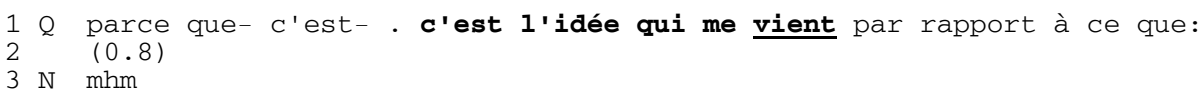

Dans cet exemple, la partie « qui me vient » ne fait que caractériser, décrire le substantif précédent, sans pour autant lui attribuer un statut focal (qui laisserait sous-entendre que c'est « l'dée » qui vient et non pas autre chose). Dans la terminologie de Lambrecht (2001 : 493), il s'agirait d'une " phrase " qui présente le focus dans la partie prédicative (predicate focus), alors que - comme nous l'avons vu - dans les clivées le focus est représenté par l'élément $X$ se situant entre $c^{\prime} e s t$ et qui/que. Dès lors, l'extrait (2) serait plus correctement interprété comme un format non marqué du type topique-focus: de ce point de vue, l'élément $c^{\prime}$ est un pronom anaphorique qui relie le prédicat (l'idée qui me vient) au discours précédent, alors que si nous avions affaire à une construction clivée, le $c^{\prime}$ ne serait pas interprétable comme un pronom anaphorique mais comme un empty subject marker (Lambrecht, 2001).

Bien que cette distinction soit justifiable dans une perspective fonctionnelle, sa pertinence peut être remise en question d'un point de vue interactionnel. En effet, si nous tenons compte du déploiement temporel des énoncés au cours de leur production dans la conversation, il n'y a aucune raison apparente de faire une différence entre les constructions clivées " canoniques " (ex. 1) et les énoncés plus fréquemment interprétés comme des phrases non marquées pragmatiquement et englobant une subordonnée (ex. 2). Au niveau formel, les deux types d'énoncés sont construits de la même manière, suivant le format c'est $X$ que/qui $Y$. Bien évidemment, on peut observer des divérsités dans le traitement discursif que les locuteurs réservent à chacune de ces constructions qui, en d'autres mots, peuvent être employées pour accomplir des tâches interactionnelles différentes. Malgré cela, d'un point de vue méthodologique la décision d'exclure de l'analyse les constructions représentées par l'exemple (2) nous semble problématique. En effet, une telle exclusion se baserait plutôt sur l'analyse " extérieure " d'un chercheur que sur l'observation de ce que les locuteurs font effectivement lorsqu'ils produisent des énoncés. Elle ne tiendrait pas compte, notamment, du déploiement progressif des tours de parole qui résulte en une grammaire constituée de manière incrémentielle. Partant, plutôt que de trancher de façon aprioristique entre les " vraies " constructions clivées et les phrases du type predicate focus, il serait nécessaire d'observer si une analyse séquentielle et interactionnelle des données nous permet d'affirmer que les participants utilisent à des fins différentes l'une et l'autre de ces constructions.

Avant de nous pencher sur l'analyse des constructions clivées dans la conversation et sur leur implication dans l'organisation des activités interactionnelles (section 4), nous présentons dans le paragraphe suivant nos réflexions sur quelques variantes de réalisation du format clivé que nous avons observé dans notre corpus. La discussion des diversités formelles contribue, à notre avis, non seulement à ouvrir le champ d'investigation à des occurrences non-standard, mais aussi à repenser fondamentalement la nature des constructions clivées. 


\section{Les variantes de réalisation}

Comme l'a remarqué entre autres Lambrecht (2001 : 493), les variantes de réalisation des constructions clivées sont beaucoup plus nombreuses que ce qui est communément décrit dans la littérature. En effet, la recherche portant sur des langues autres que l'anglais et le français a montré qu'il existe un grand nombre de variantes formelles : nous pouvons citer - pour rester dans le domaine des langues romanes - le cas de l'italien, étudié sous cet aspect notamment par Frison (1988) et par Berretta (1994) qui dressent un riche inventaire des variantes attestées. ${ }^{4}$ Ce travail reste à faire pour le français : c'est pourquoi nous allons décrire aux paragraphes suivants quelques-uns des formats récurrents dans le corpus soumis à examen. Seront exposées d'abord les formes dont on trouve mention dans les études antérieures - par exemple les variations concernant l'élément se trouvant dans la position $X(2.1)$ ou le cas des formes introduites par une expression présentative négative du type $c(e n)^{\prime}$ est pas (2.2). Nous nous pencherons ensuite sur des occurrences qui n'ont pas fait l'objet, à ce jour, d'une description détaillée et qui portent sur certains éléments de la clivée que les définitions traditionnelles considèrent comme constitutifs de ce type de construction syntaxique, comme la présentative initiale (2.3) et l'élément connecteur (2.4).

\subsection{Un lieu de variation : l'élément $X$}

Dans la construction $c^{\prime} e s t X$ que/qui $Y$, différentes sortes d'unités langagières peuvent se situer dans la position indiquée par un $X$. S'appuyant largement sur des études menées sur la langue anglaise, les exemples discutés dans la littérature présentent la plupart du temps des énoncés dans lesquels cette position est occupée par un syntagme nominal (lexical), comme dans le cas suivant :

(3) corpus B, 1254-1255

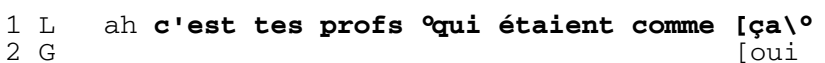

Si en anglais c'est effectivement très souvent un syntagme nominal qui prend la place de l'élément $X$, le corpus français soumis à examen ne contient que de très rares occurrences de ce genre. Cette constatation nous oriente vers un problème qui se manifeste dans une partie de la recherche linguistique sur les constructions clivées : en effet, celle-ci est souvent conduite sur la base d'exemples inventés ; c'est le cas, du moins, des articles les plus cités (cf. Lambrecht, 2001 ; Katz, 2000 ; Geluykens, 1988). Dans ces études, les constructions clivées contenant un syntagme nominal lexical en position $X$ sont souvent traitées comme des formes " canoniques" de cette structure syntaxique. L'analyse de nos données empiriques a révélé toutefois que - du moins dans notre corpus - cette position est plus souvent occupée par des unités appartenant à d'autres catégories grammaticales.

L'exemple (3) nous permet toutefois de mettre en évidence un aspect des formats clivées qui se révèle être pertinent pour le traitement de ce genre d'énoncés en termes de " constructions ": on observe dans cet extrait qu'il n'y a pas d'accord de nombre entre l'élément c'est et le référent tes profs. Ce phénomène récurrent en français parlé - a été interprété par Lehmann (à paraître) comme un indice qui rendrait visible un processus de grammaticalisation en cours : par conséquent, l'élément $c^{\prime} e s t$ se révèle être une composante de la construction qui est en voie de figement. Le même phénomène est également observable dans l'extrait (1), où le c'est est suivi d'un constituant au pluriel. ${ }^{5}$ L'auteur entrevoit un indice ultérieur qui témoignerait d'un processus de grammaticalisation: il constate en effet que dans de nombreux cas, les constructions présentant une syntaxe clivée ont perdu leur effet pragmatique de focalisation ou de contraste. C'est une observation qui justifie, entre autres, notre décision de tenir compte dans l'analyse aussi des occurrences présentant une structure syntaxique du type " clivée " qui ne résulte pas forcément dans une mise en valeur de l'élément occupant la position $X$.

Parmi les unités grammaticales qui peuvent prendre la place de l'élément $X$ nous trouvons également des adverbes, comme dans l'exemple suivant :

(4) corpus A, 2633-2635

$1 \mathrm{Q}$ ah on recommence encore une fois/

2 B puisque avant on était en orientation donc là . non \non \c'est MAINTENANT qu'on 
Nous pouvons affirmer avec Nølke (1983) que les adverbes temporaux (mais aussi d'autres types d'adverbes) apparaissent de manière récurrente dans les constructions clivées en français. Occupant la position focale, ils peuvent également être marqués par des moyens prosodiques : ce phénomène - que Lambrecht (2001) décrit comme étant caractéristique de toutes les constructions clivées - est observable dans l'extrait (4), où l'on observe une proéminence prosodique sur la première syllabe de «MAINTENANT », mot qui, de surcroît, est prononcé avec une haute amplitude de la voix. Notons au passage qu'on marquage prosodique est également observable dans l'exemple (3), où la deuxième partie de l'énoncé (commençant par le pronom relatif « qui ») est prononcé à voix basse.

De nombreuses autres unités grammaticales émergent de façon récurrente dans la position entouré par l'élément présentatif $c^{\prime}$ est et le pronom relatif que/qui. Ainsi nous observons avec une certaine fréquence que cette position peut être occupée par des syntagmes prépositionnels, comme nous l'expliquerons au paragraphe suivant.

\subsubsection{La position $X$ englobant une préposition}

Différents chercheurs travaillant sur le français se sont penchés sur les " compléments prépositionnels » (Blanche-Benveniste, 2001b : 85) qui peuvent se situer dans celle qui est perçue comme la partie focale d'une construction clivée - mais qui peuvent également être combinés avec l'élément connecteur que/qui : ainsi, Muller 2003 : 102 distingue trois types de phrases clivées : le moderne (C'est à ma mère que tu as parlé), l'ancien (C'est ma mère à qui tu as parlé) et le redondant (C'est à ma mère à qui tu as parlé). ${ }^{6}$ L'auteur justifie cette terminologie en avançant d'abord un critère de fréquence et ensuite un argument formel : le type ancien serait moins fréquent que le type moderne en français contemporain; quant au type redondant, il se caractériserait par un double marquage de l'objet indirect. Les extraits tirés de notre corpus montrent que le type moderne est en effet amplement utilisé par les locuteurs, alors que les variantes ancienne et redondante sont peu attestées. En revanche, nous constatons que lorsque la position $X$ est introduite par une préposition, celle-ci est rarement constituée de l'élément à. Il s'agit d'une préposition que Rouquier, 2007: 192 qualifie de «légère », catégorie que l'auteure applique aussi à l'élément de et qu'elle oppose aux prépositions «sémantiquement lourdes » comme par et sur. L'examen systématique de notre corpus a révélé que les prépositions appartenant à cette deuxième catégorie étaient bien plus fréquentes que les unités à et de dans les constructions clivées. Voici quelques exemples :

(5) corpus D, 2011

1 D c'est pour ça que moi je dis je navigue entre les deux choses\}

L'exemple atteste d'un usage récurrent dans le parler interactionnel : comme nous le montrerons au paragraphe 3, la construction clivée c'est pour ça que $Y$ introduit souvent une conclusion, une synthèse de ce qui a été dit auparavant. L'expression - que Lambrecht, 2001 : 501 mentionne en passant sans pour autant proposer une analyse détaillée - apparaît avec une fréquence considérable dans notre corpus, au point que nous tendons à y reconnaître une expression désormais lexicalisée ou du moins en cours de lexicalisation.

Nous avons également repéré d'autres prépositions qui interviennent toutefois avec une fréquence mineure. Ainsi, les exemples suivants témoignent d'occurrences isolées :

(6) corpus I, 1678

$1 \mathrm{~F}(\ldots)$ comme si c'est avec ces arguments-là qu'on pouvait racole:r/ [...]

(7) corpus I, 1785

1 F l'allemand/ C'est par là qu'on te tient $\backslash$

(8) corpus B, 2094

1 L C'est de là que ça vient 
Comme nous l'avons vu aux paragraphes précédents - et contrairement à ce que soutiennent les descriptions traditionnelles des constructions clivées - la position $X$ ne comprend pas nécessairement un nom ou un pronom (comme c'est le cas par exemple dans l'extrait (6)). En effet, les exemples (7) et (8) montrent des occurrences dans lesquelles l'élément $X$ est constitué d'une préposition suivie d'un adverbe de lieu. Notons que cette possibilité n'est pas prise en considération par Nølke (1983) qui admet parmi les adverbes pouvant intervenir dans des constructions clivées uniquement des adverbes de temps et des adverbes " paradigmatisants ". ${ }^{7}$ Cependant, malgré l'absence d'un terme nominal dans la position $X$, les deux exemples (7) et (8) peuvent être analysés raisonnablement comme des constructions clivées aussi dans une perspective strictement fonctionnelle : dans les deux cas la construction syntaxique permet en effet de mettre en avant (de focaliser) la partie initiale de l'énoncé.

\subsection{La négation}

Une construction clivée peu explorée jusqu'ici mais également courante dans la langue parlée se caractérise par la négation de l'élément présentatif :

(9) corpus F, 731-732

$1 \mathrm{~V}$ ouais c'est pas des mots qu'on ut-que: : (qu-) très compliqués . qu'on utilise

Dans cet exemple, on perçoit la deuxième fonction pragmatique communément attribuée aux constructions clivées, c'est-à-dire leur capacité d'exprimer une opposition, un contraste. L'effet contrastif repose en grande partie sur la catégorisation des « mots » comme étant « très compliqués », ce qui laisse sous-entendre l'existence de mots qui serait peu ou moins compliqués. Cette catégorisation est réalisée quant à elle à l'intérieur de la position $X$, à savoir entre le « c'est pas » initial et l'élément « qu' ».

L'exemple suivant rend compte d'un autre usage fréquent en français parlé, qui présente certaines caractéristiques formelles des constructions clivées :

(10) corpus I, 506

$1 \mathrm{~F}$ c'est pas parce que ce déclic se produit qu'y aura plus de fautes/ (...)

Quelques exemples similaires ont été décrits par Lambrecht, 2001 : 495-496 qui renvoie à l'interprétation idiomatique à laquelle ces énoncés donnent lieu dans la plupart des cas. Du point de vue de l'auteur, la partie focale ne se trouve pas dans l'espace encadré par «c'est pas » et «que » (ce n'est donc pas le segment «parce que ce déclic se produit»), mais dans la partie qui suit le «que ». En effet, l'énoncé peut être interprété de différentes manières, selon qu'il est perçu comme une expression idiomatique ou une construction clivée : dans la première interprétation, l'assertion du locuteur est qu'il y aura davantage de fautes, même si un déclic se produit. La deuxième lecture de l'énoncé révèle un sens très différent : dans cette optique, le locuteur affirmerait qu'il n'y aura plus de fautes et que la raison pour cela n'est pas liée au fait qu'un déclic se produise. Bien que les deux interprétations de cet énoncé soient effectivement possibles, la lecture idiomatique semble prévaloir dans l'usage. Toutefois, d'un point de vue interactionnel, cette explication ne permet pas d'exclure de l'analyse des occurrences de ce genre: l'interprétation que les locuteurs font d'un tour de parole est exhibée, négociée, précisée etc. au cours du déploiement de l'échange conversationnel. D'un point de vue formel, la structure syntaxique de l'énoncé (10) présente effectivement de fortes similitudes avec les constructions clivées " canoniques ". On y reconnaît une structure bipartite qui pourrait toutefois se distinguer de celle qu'on observe communément dans les constructions clivées. Le patron de base (c'est pas parce que $X$ que $Y$ ) révèle en effet deux différences formelles : d'une part, la partie initiale " stable" est plus élaborée (c'est pas parce que à la place du simple $c^{\prime}$ est dans les constructions clivées classiques), d'autre part l'élément connecteur entre les positions $X$ et $Y$ semble être limité à l'unité que, alors que dans les autres constructions clivées celui-ci peut être réalisé par d'autres pronoms relatifs, comme qui et où (cf. section 2.4.). 


\subsection{L'absence du c'est}

La variabilité des constructions clivées peut intéresser aussi des éléments qui sont traditionnellement décrits comme constitutifs de cette structure syntaxique. Ainsi, l'expression présentative initiale est considérée comme obligatoire dans les descriptions traditionnelles. Pourtant, les locuteurs produisent aussi des énoncés comme le suivant :

(11) corpus B, 533-539

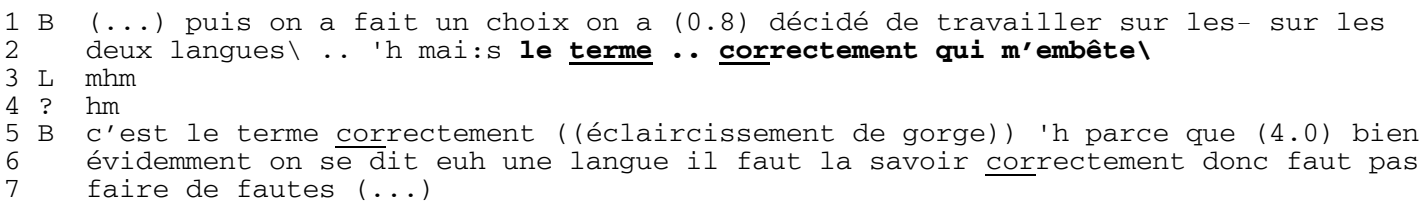

L'extrait reproduit la fin potentielle du tour de parole de B (1. 2) - reconnaissable aussi à l'intonation descendante de «m'embête $»$ et aux signes d'accord qui suivent (1l. 2 et 3). Précédemment, les participants discutaient d'une définition du bilinguisme, selon laquelle la personne bilingue serait celle qui parle correctement deux langues. A la ligne 2, B arrive à une fin de tour possible et rend cette fin reconnaissable, entre autres, par une structure focalisante dans laquelle l'expression «le terme .. correctement » est mise en évidence par des indices prosodiques, le mot «terme » étant accentué et, en outre, suivi d'une pause. De même, la première partie de l'adverbe "correctement» est elle aussi accentuée. Le deuxième élément qui contribue à configurer mise en évidence du mot « terme » est réalisé par l'élément «qui » qui rend possible, rétrospectivement, une interprétation de la structure syntaxique. La fin du tour de parole de B (1. 2) semble donc réaliser les effets pragmatiques que l'on attribue communément aux constructions clivées, alors que celle-ci a été produite sans le c'est initial que les livres de grammaire prévoient. Il est toutefois intéressant de constater que B est visiblement orienté vers le format " traditionnel " de la clivée. En effet, après des signes d'accord de la part de ses interlocuteurs (1l. 3 et 4), B reprend la parole et renoue avec son tour précédent : il le fait en affirmant que " c'est le terme correctement» (1. 5), ce qui lui permet, entre autres, de mettre en évidence pour la deuxième fois l'argument central de son énoncé précédent.

\subsection{L'expression de la connexion}

La variation s'exprime aussi dans l'élément qui marque la connexion entre la partie présentative de la construction clivée et la clause relative avec laquelle elle forme une unique proposition. L'une des variantes les plus fréquentes est visible dans l'exemple suivant :

(12) corpus B, 2953-2954

$1 \mathrm{~N} \quad \mathrm{C}^{\prime}$ :est $\mathrm{C}^{\prime}$ : est là où je suis ... de nouveau un peu plus . tolérant parfois

2 j'ai: . j'ai une dent contre les langues

L'élément de connexion est formé ici du pronom relatif «où » qui est motivé à son tour par la nature grammaticale de l'élément qui lui précède, à savoir l'adverbe de lieu « là ». De manière intéressante, la construction là où est souvent analysée comme exemple d'un clivage redondant (Blanche-Benveniste, 2001b : 86 ; Muller, 2003 : 106-17) qui - comme nous l'avons mentionné plus haut (2.1.1) - se caractérise par la double présence d'une préposition à la fois devant l'élément nominal et devant l'unité que/qui. Le seul trait qui permet de réunir l'occurrence que nous observons dans l'exemple (12) avec les constructions clivées redondantes est représenté par le double marquage, non pas d'une préposition, mais d'un adverbe de lieu. Bien évidemment, il s'agit dans ce cas d'une utilisation lexicalisée de l'adverbe «là » qui ne renvoie pas à un endroit extralinguistique mais dont la référence reste plutôt vague. ${ }^{8}$ Cependant, l'emploi de « où »-qui fonctionne ici comme un pronom relatif - n'est pas attesté de façon systématique. En effet, une vingtaine de secondes plus tard, le même locuteur produit l'énoncé suivant :

(13) corpus B, 2978-2981

$1 \mathrm{~N}$ et C'est là que j'ai parfois un- .. une frustration (1.0) très 


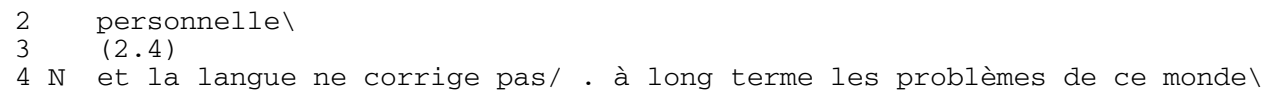

Au-delà de la discussion sur la variation entre où et que, on peut se demander si les exemples (12) et (13) peuvent effectivement être considérés comme des constructions clivées. Si d'un point de vue formel on y reconnaît facilement la structure canonique du format clivé (c'est X que/qui Y), l'analyse fonctionnelle nous ne permet pas de corroborer, avec autant de certitude, la fonction focalisante que l'on a l'habitude d'attribuer aux constructions clivées : selon ce point de vue, l'unité « là » constituerait en effet dans les deux occurrences le focus de la phrase. Il semblerait que dans les constructions utilisées dans les exemples (12) et (13) l'effet focalisant soit affaibli par le caractère idiomatique de l'expression: la fréquence des formules c'est là où/c'est là que est en effet telle que nous pouvons y reconnaître des formes figées. Toutefois, cela ne devrait pas nous empêcher de tenir compte de réalisations de ce genre, lorsque nous analysons la manière dont les interlocuteurs font intervenir les constructions clivées dans leurs interactions. Comme nous le verrons dans les sections 3.2 et 3.3, nous avons rencontré dans notre corpus plusieurs cas de constructions clivées utilisées comme des formules figées et intervenant tout de même dans l'organisation séquentielle de l'interaction.

\section{De la phrase clivée à la construction clivée}

Nous avons vu aux paragraphes précédents que les descriptions traditionnelles des constructions clivées se heurtent aux nombreuses variations avec lesquelles celles-ci apparaissent dans les données conversationnelles. Les linguistes qui ont essayé d'intégrer ces variations dans leurs modèles d'analyse ont souvent cédé à la tentation de créer des catégorisations sophistiquées, en tenant compte des différents formes dans lesquelles se manifestent les structures clivées: ainsi, Clech-Darbon, Rebuschi \& Rialland (1999) identifient quatre types de constructions clivées - se différenciant aux niveaux de la nature grammaticale et de l'extension de l'élément focal - qui accomplissent des fonctions discursives distinctes (pour une autre taxonomie des constructions clivées cf. Rouget \& Salze, 1985). Le couplage formefonction, si souvent évoqué pour les constructions clivées, pose toutefois un certain nombre de problèmes : ainsi, les choix formels des locuteurs ne sont pas simplement issus de contraintes d'ordre syntaxique ou informationnel, mais contribuent à l'organisation interactive du discours, dans ses dimensions à la fois grammaticales, séquentielles et interactionnelles. La fonction d'un format syntaxique n'est pas inscrit dans sa forme, mais elle émerge de l'utilisation située que les participants font des ressources langagières. Dans une perspective comparative, certains auteurs ont souligné que les constructions clivées n'accomplissaient pas nécessairement les mêmes tâches discursives dans des langues différentes, par exemple en français et en anglais (Katz, 2000 ; Lambrecht, 2001 : 492). Ce sont des arguments qui remettent en question l'exclusion de certaines structures syntaxiques - qui d'un point de vue fonctionnel ne sont habituellement pas considérées comme des clivées, même si elles présentent des réalisations formelles similaires. Il convient alors plutôt de considérer les structures clivées comme des constructions au sens de la grammaire constructionnelle (Construction Grammar). C'est surtout dans l'approche dite usage based (Croft, 2001 ; Deppermann, 2006) que les constructions sont perçues non pas comme des structures syntaxiques qui respectent certaines règles grammaticales, mais plutôt comme des gestalt qui peuvent avoir différentes réalisations formelles. Ainsi, on observe de façon récurrente dans les données empiriques que les constructions sont réalisées de façon fragmentaire (v. aussi l'ex. (11)). Souvent, ces fragments ne peuvent pas être attribués à une construction schématique bien précise. C'est pour cette raison qu'Imo (2007: 38$)$ propose de traiter les constructions comme des prototypes et de considérer les réalisations de ces constructions - qu'il appelle constructs ou tokens - comme des représentants des prototypes abstraits qui eux ne se réalisent jamais dans leur forme parfaite. C'est une position qui rejoint la conception de la grammaire émergente de Paul Hopper : ce linguiste considère la langue comme un inventaire de constructions que les locuteurs utilisent dans le discours (cf. Hopper, 2004). La plupart du temps celles-ci n'apparaissent pas, dans la conversation spontanée, comme des unités complètes, mais comme des fragments de constructions qui sont combinés avec d'autres fragments. Dans sa manière de voir, la grammaire n'est par conséquent qu'un épiphénomène de combinaisons fréquentes des mêmes structures. Cela signifie que la grammaire n'est pas une précondition nécessaire qui rend 
possible l'interaction. Elle est considérée plutôt comme résultant des interactions sociales et s'inscrivant dans un processus continuel d'adaptation locale (d'où l'idée de l'émergence). Ainsi, la structure pseudoclivée est souvent réalisée uniquement à travers la partie initiale de sa forme canonique (Hopper, 2004) et utilisée, dans l'interaction, pour introduire un tour de parole long (Hopper, 2004 ; Müller, à paraître). Par rapport à la construction clivée, Hopper \& Thomspon (à paraître) soulignent que la première partie de la clause est relativement fixe ( $c^{\prime} e s t$... que), alors que la suite est très ouverte. Dans cette perspective, les constructions sont donc perçues comme des parties formulaïques que les locuteurs utilisent pour organiser leur discours, alors que les formes que l'on trouve dans les textes littéraires seraient des versions normativisées et étendues des formules utilisées dans la langue parlée.

Les réflexions qui se sont développées au sein des approches récentes de la langue parlée témoignent de la difficulté qui émane de la description des constructions syntaxiques en termes transformationnels, comme lorsque l'on présume l'existence d'une clause canonique du type Sujet-Verbe-Objet qui permettrait d'en dériver des variantes clivées. Plutôt que d'essayer de décrire les procédures mentales, transformationnelles qui seraient mises en œuvre lors de la production d'un énoncé clivé, nous baserons nos analyses sur les activités observables des interlocuteurs. Dans cette perspective, les énoncés des locuteurs ne sont pas perçus comme émanant de " pré-formes " canoniques, mais comme des productions qui répondent aux contingences de la conversation en cours.

\section{Les aspects interactionnels}

Au-delà des nombreuses études portant sur la structure informationnelle des constructions clivées, les recherches traitant de la manière dont celles-ci interviennent dans la gestion du discours sont rares. Ainsi, les articles annonçant une étude du fonctionnement discursif des constructions clivées se limitent bien souvent à analyser (certes de manière très détaillée) les éléments focaux et topicaux de ces constructions et à déterminer leur accessibilité dans le contexte discursif (cf. Hedberg, 1990 ; Geluykens, 1991). Dans une perspective alternative, Delin (1990) et Delin \& Oberlander (1995) montrent que les constructions clivées peuvent contribuer à structurer certains aspects du discours qui relèvent de la gestion de l'interaction. En l'occurrence, Delin (1990) décrit trois tâches que les constructions clivées accomplissent de manière régulière dans le corpus (anglais écrit et conversationnel) que la chercheuse a exploré : a) correction move: cette fonction - qui rappelle l'emploi contrastif traditionnellement attribué aux constructions clivées - permet de corriger, de " réparer" un énoncé ou une partie d'un tour de parole précédent ; b) continuation move : souvent, les constructions clivées permettent aux locuteurs de marquer la " continuation " d'un tour de parole, ou, en d'autres termes, d'exhiber qu'ils sont en train de développer un énoncé étendu ; c) fill move : les constructions clivées peuvent intervenir également dans la deuxième partie d'une paire adjacente du type question/réponse. Ainsi, à une question du type Qui a pris le pain?, il est possible de répondre par C'est moi qui ai pris le pain (exemples inventés), même si dans notre corpus nous n'avons pas observé des occurrences de ce genre. Bien que l'approche qu'adoptent Delin (1990) et Delin \& Oberhauser (1995) parcoure des chemins orientés vers les aspects interactionnels, elle reste ancrée dans une vision informationnelle - et donc transformationnelle - des constructions clivées. ${ }^{9}$ Afin de mieux dégager les tâches que les constructions clivées accomplissent dans les échanges conversationnels, nous analyserons dans les paragraphes suivants le traitement interactionnel que les locuteurs eux-mêmes réservent aux constructions clivées. Nous verrons d'abord que le caractère bipartite que nous avons évoqué plus haut ne repose pas sur une analyse introspective, mais que ce sont les locuteurs eux-mêmes qui, par leurs comportements conversationnels, rendent possible une telle interprétation (4.1). Nous montrerons ensuite qu'en utilisant une construction clivée les locuteurs ne structurent pas uniquement le niveau informationnel de la phrase, mais que celle-ci est employée également pour organiser le discours au-delà du tour de parole dans lequel elle apparaît. L'analyse systématique de notre corpus a dévoilé que les locuteurs accomplissaient deux tâches bien différentes en recourant à une construction clivée : d'une part, elle permet d'introduire un tour de parole étendu (4.2), d'autre part de clore un épisode conversationnel (4.3). 


\subsection{Le caractère bipartite de la construction clivée}

Les linguistes qui s'intéressent aux constructions clivées appuient leurs analyses généralement à l'aide de clauses complètes qui sont soit des phrases tirées d'un texte écrit soit des productions verbales attestées ou construites par introspection. Déjà dans les premières recherches sur les clivées, celles-ci ont été décrites comme des unités cohérentes qui ne peuvent pas être interrompues par des pauses (Jespersen, 1937: 84) et qui se caractérisent par une proéminence prosodique sur l'élément focal (Lambrecht, 2001). L'examen des données conversationnelles montre toutefois que les constructions clivées ne sont pas toujours réalisées " en bloc ". En attestent, par exemple, des cas où la première partie de la clivée est séparée de la partie finale par un tour de parole de l'interlocuteur, comme dans l'extrait suivant. Les participants viennent d'évoquer le cas d'une étudiante qui, en tant que stagiaire, a donné des cours d'italien dans une classe de lycée. L'un des locuteurs a assisté à ces cours et vient d'expliquer que l'étudiante enseignait un italien très littéraire :

\section{(14) corpus A, 1568-1576}

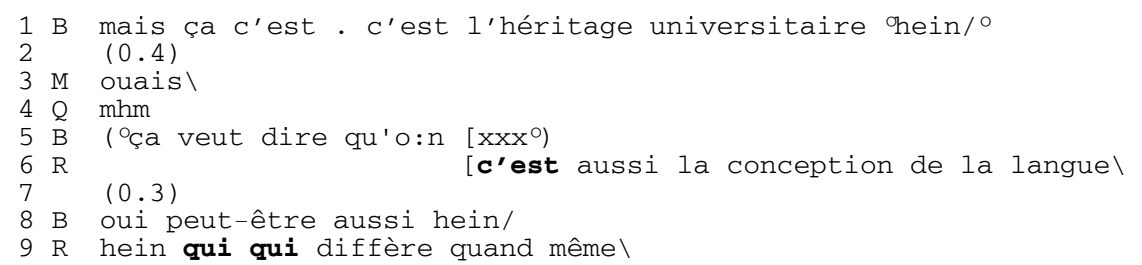

A la ligne 1, B produit un énoncé par lequel il formule une explication relative à la méthode d'enseignement de l'étudiante discutée précédemment. Celle-ci est suivie d'une pause (1. 2) et de deux signes d'accord réalisés par deux participants différents (11. 3-4). En chevauchant un tour de B (1. 5), le locuteur $\mathrm{R}$ prend la parole à la ligne 6 et formule une deuxième explication, en initiant son tour de parole par l'élément $c^{\prime}$ est et en reprenant ainsi le format syntaxique que $\mathrm{B}$ avait utilisé à la ligne 1 . L'énoncé de $\mathrm{R}$ est perçu comme un tour de parole achevé, comme on le voit à la ligne 8 , où $\mathrm{B}$ s'autosélectionne en ratifiant la position que $\mathrm{R}$ vient d'exprimer. De manière intéressante, $\mathrm{R}$ reprend la parole à la ligne 9 en rattachant sa production verbale au tour de parole qu'il avait produit auparavant (1.6) et en donnant lieu, de cette manière, à la réalisation d'une construction clivée. Cet ajout - qui peut être décrit comme un incrément (Schegloff, 1996) - comporte, rétrospectivement, le recadrage du tour produit à la ligne $6:$ si dans un premier temps celui-ci était exhibé et traité (par B) comme un tour de parole achevé tant au niveau syntaxique que sur les plans pragmatique et prosodique, l'ajout de la ligne 9 le transforme en une première partie d'une construction clivée. En d'autres termes, bien que B exhibe par son intervention (1. 8) son orientation vers la complétude du tour précédent, l'incrément par lequel R enchaîne (1. 9) permet le reformatage grammatical d'un tour " possiblement complet ". Dès lors, cet exemple montre d'une part que la grammaire utilisée pour l'interaction ne peut pas être conçue comme un ensemble de règles figées que les locuteurs actualiseraient au fur et à mesure que la conversation avance : au contraire, les structures syntaxiques sont continuellement adaptées aux besoins interactionnels et situés des participants. D'autre part, l'extrait nous permet d'observer la structure bipartite de la construction clivée : c'est à travers ses énoncés (1l. 6 et 9) que R rend visible la manière dont lui-même analyse ses propres productions verbales.

Le caractère bipartite des constructions clivées émerge aussi de l'extrait suivant, dans lequel G est en train de démontrer les avantages que des compétences bilingues peuvent acquérir dans le monde professionnel :

\section{(15) corpus C, 1673-1676}

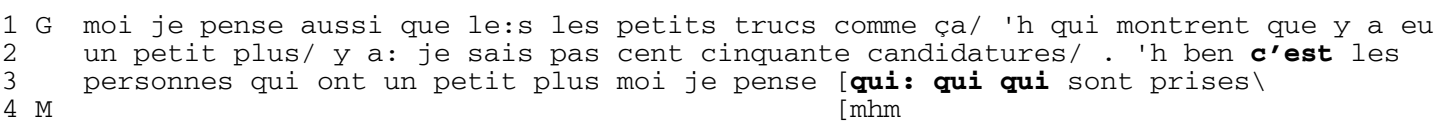

Cet extrait montre d'autres manières de s'orienter vers le format syntaxique de la construction clivée, tant du côté de la locutrice en cours $(\mathrm{G})$, tant par la participation qu'exhibe son interlocutrice (M). Ainsi, on observe que $\mathrm{G}$ introduit une formule idiomatique («moi je pense », 1. 3) immédiatement après la fin de la 
première partie de la construction clivée initiée à la ligne 2. Se faisant, elle insère une expression parenthétique après la première partie $\left(c^{\prime} e s t X\right)$ de la construction clivée. L'analyse de nos données a montré de manière récurrente que c'est à ce moment précis que les locuteurs peuvent suspendre la réalisation de la construction clivée. De la part de l'interlocutrice, nous observons qu'elle aussi exhibe sa manière de segmenter le tour de parole de $G$ : elle formule en effet un signe d'accord («mhm », 1. 4) en chevauchement avec le premier «qui: » de G (1. 3) qui introduit la deuxième partie de la construction clivée.

L'analyse des extraits (14) et (15) a révélé que le traitement des constructions clivées comme des structures syntaxiques bipartites ne repose pas principalement sur le regard que le linguiste apporte sur les données d'un point de vue extérieur : ce traitement découle plutôt de la manière dont les participants à la conversation eux-mêmes traitent les formats syntaxiques en question. En outre, la discussion des exemples nous a permis de voir le caractère processuel de la grammaire telle qu'elle est mise en œuvre dans la conversation. La grammaire constitue en effet une ressource qui se déploie de façon incrémentielle dans les échanges verbaux et que les participants adaptent continuellement aux contingences interactionnelles de la conversation en cours. De ce point de vue, la grammaire ne peut pas être perçue comme un ensemble de règles plus ou moins stables que les locuteurs actualiseraient dans le discours : il s'agit plutôt d'une ressource qui est sensible à la structuration temporelle et séquentielle de l'interaction et qui, de ce fait, se caractérise par une très grande flexibilité (cf. Auer, 2005 qui parle à ce propos d'on-line syntax).

\subsection{La structuration d'un tour de parole long}

Dans le corpus soumis à examen, les constructions clivées sont souvent impliquées dans la structuration de tours de parole étendus. Dans l'exemple suivant, le locuteur Q décrit la situation linguistique du Val d'Aoste en précisant le rôle que joue le gouvernement régional en matière de bilinguisme :

(16) corpus B, 3236-3241

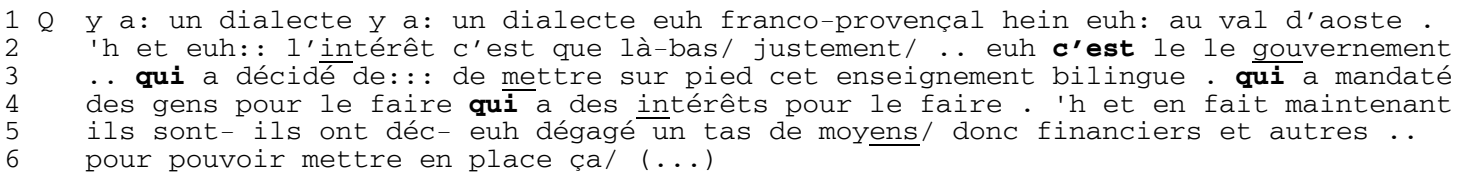

On observe une construction pseudo-clivée dans la partie initiale du tour (« l'intérêt c'est que », 1. 2) qui selon les recherches de Müller (à paraître) est souvent utilisée pour initier un tour de parole étendu. Dans le cas présent, l'extension du tour est également accomplie par une construction clivée. A la ligne 2, Q s'oriente vers cette structure à travers les mots «c'est le le gouvernement ». Après une micropause (1. 3) il poursuit son discours en faisant suivre trois clauses relatives dont chacune est rattachée - syntaxiquement et sémantiquement - au syntagme nominal « le gouvernement ». Nous observons donc une répétition de la deuxième partie de la construction clivée qui contribue à prolonger le tour de parole et à introduire des éléments thématiques qui vont être développés dans la continuation du tour. Dans ce cas aussi, le locuteur exploite visiblement le format bipartite de la construction clivée.

Dans l'exemple qui suit, on observe une construction clivée très récurrente dans notre corpus. Les interlocuteurs sont en train de discuter de deux définitions du terme " bilinguisme " que l'enquêtrice (Q) vient de leur présenter :

(17) corpus C, 419-432

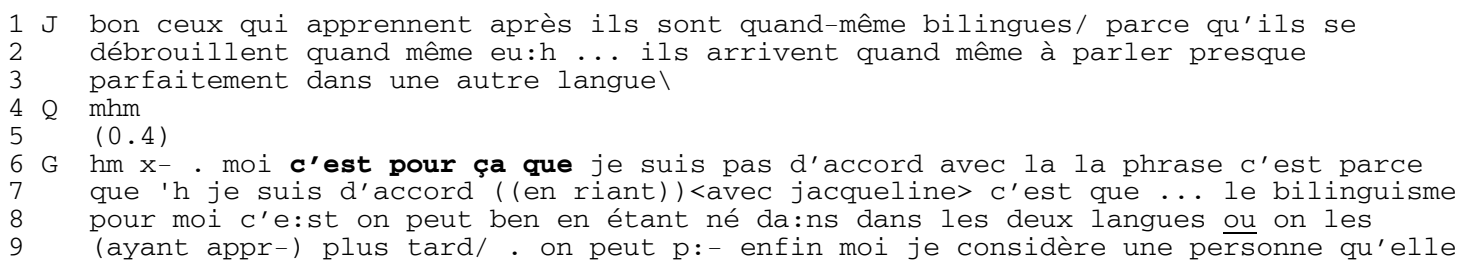




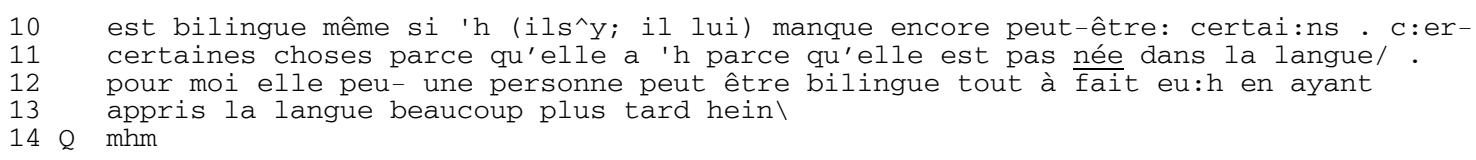

Aux lignes 1-3 J termine son intervention en précisant qu'une personne ayant appris une deuxième langue dans un contexte scolaire peut aussi être considérée comme bilingue. Après un signe d'accord de la part de $Q(1.4)$ et une pause brève (1. 5), la locutrice G s'autoselectionne et introduit peu après la construction clivée qui commence par les mots «c'est pour ça que » (1.6). Cette expression - qui dans l'usage actuel introduit une explication, une justification etc. - inaugure ici un tour de parole long par lequel $\mathrm{G}$ montre son accord avec ce que $\mathrm{J}$ vient de dire, tout en mettant en évidence que l'accord repose sur une conviction personnelle de $G$ (cf. l'emploi fréquent des pronoms «moi» et «je»). Cette explication s'achève seulement à la 1.13 , comme le montre la tag question « hein » en fin de tour et le signe de ratification que réalise $\mathrm{Q}$ à la 1.14 .

Si les deux derniers extraits nous ont permis de constater l'implication des constructions clivées dans l'organisation des tours de parole, ils nous orientent aussi vers une problématique qui est inhérente à la recherche traditionnelle sur les constructions clivées, s'inscrivant dans une vision transformationnelle et analysant les constructions délimitées en clauses. En particulier, l'adoption d'une perspective phrastique ne permet pas d'étudier l'émergence des constructions clivées telles qu'elles sont effectivement utilisées par les locuteurs. De surcroît, la recherche se basant sur des phrases isolées (et souvent décontextualisées) ne permet pas de tenir compte de la manière dont les locuteurs reformatent continuellement leurs tours de parole, donnant ainsi lieu à des structures syntaxiques qui ne sont pas analysables en termes de " phrases " ou de " clauses ", comme l'a prouvé l'analyse de l'extrait (17).

\subsection{La clôture d'épisodes conversationnels}

De manière intéressante, l'expression c'est pour ça que $Y$ - que nous venons d'analyser comme une ressource qui permet d'introduire un tour de parole étendu - est également utilisée pour accomplir la tâche opposée, c'est-à-dire pour clore un tour de parole. Ce fonctionnement est clairement visible dans l'extrait suivant, dans lequel le locuteur N précise qu'il n'est « plus tellement bilingue » :

(18) corpus D, 1966-1977

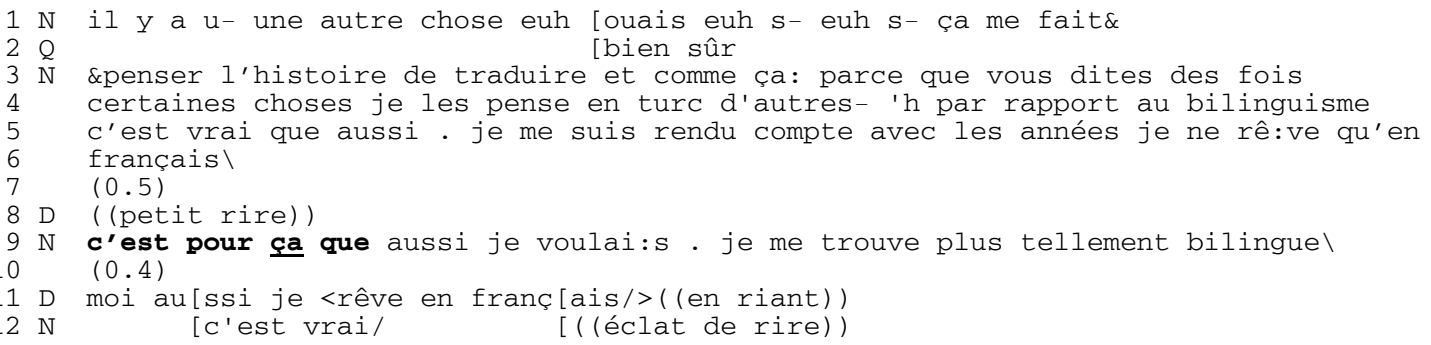

A la ligne $6 \mathrm{~N}$ arrive à une première fin de tour qui donne lieu à un point de transition possible, le tour de $\mathrm{N}$ étant syntaxiquement et prosodiquement complété (cf. l'intonation descendante sur « français \», 1. 6). Aucun autre locuteur ne prend la parole à ce moment précis, comme le montre la pause de 0,5 secondes qui a lieu à la ligne 7. Après un petit rire de la part de D (1. 8) N s'autosélectionne à la ligne 9 et produit un énoncé qui marque la fin de son raisonnement. Cette tâche est réalisée à l'aide d'une construction clivée (« c'est pour ça que aussi je voulai:s . je me trouve plus tellement bilingue \», 1. 9) qui - dans ce cas précis - signale la fin d'un épisode conversationnel. Jusqu'ici c'était N qui exposait ses expériences avec le bilinguisme, alors qu'à partir de la ligne 10 c'est $\mathrm{D}$ qui parlera de ses pratiques langagières. Il est intéressant de constater que c'est seulement à la fin de la construction clivée qu'un autre locuteur prend la parole - alors que le changement de locuteur ne s'est pas vérifié précédemment, par exemple pendant la pause de 0,5 secondes survenue à la ligne 7 . Cela ne signifie bien évidemment pas que les interlocuteurs auraient attendu l'émergence d'une construction clivée avant de reprendre la parole. Cela montre toutefois 
que ce sont les participants mêmes (en l'occurrence le locuteur D) qui reconnaissent une ressource de clôture dans la construction clivée telle qu'elle est employée par N. L'argument que nous avançons ici ne consiste pas à dire que la construction clivée fonctionnerait, à elle seule, comme un dispositif de clôture. Il est évident que d'autres aspects co-occurrents contribuent à orienter en ce sens le tour de parole final de $\mathrm{N}$. En conséquence, le caractère formulä̈que de l'expression c'est pour ça que $Y$ ainsi que son implication fréquente dans des séquences d'explication contribuent à exhiber l'orientation vers l'abandon du floor conversationnel de la part de N.

Le fait que les constructions clivées puissent être utilisées pour rendre reconnaissable la fin d'un épisode conversationnel est visible aussi à travers une autre tâche que celles-ci accomplissent de manière récurrente. En effet, lorsque les clivées sont utilisées pour exprimer un raisonnement final, une constatation conclusive etc., elles sont systématiquement suivies d'un signe d'accord de la part d'un interlocuteur. De ce point de vue, elles constituent des ressources qui permettent de solliciter un accord de la part d'un interlocuteur, comme le montre le dernier extrait que nous analyserons ici :

(19) corpus C, 1119-1131

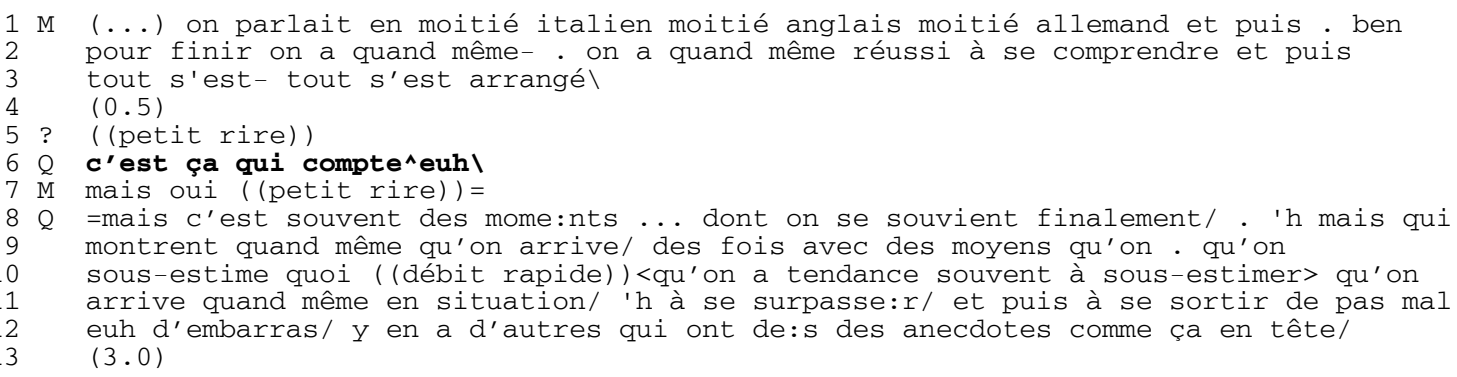

Dans cet exemple, l'énoncé de la locutrice M se termine par une formule généralisante («puis tout s'esttout s'est arrangé \», 1. 3) qui clôt l'activité narrative dans laquelle elle s'était engagée jusqu'à ce moment. Les interlocuteurs rendent également reconnaissable la fin du tour en intervenant de différentes manières : un locuteur non identifiable produit un petit rire à la 1.5 , alors que tout de suite après, Q formule un énoncé évaluatif («c'est ça qui compte^euh », 1. 6) lui permettant de donner une appréciation finale de ce que $\mathrm{M}$ vient de dire. Le tour de $\mathrm{Q}$ - qui est interprétable comme une construction clivée - projette un signe d'accord de la part de $\mathrm{M}$, qui suit effectivement à la ligne 7 («mais oui ((petit rire)) »). Cette manière de structurer la fin d'un épisode narratif est bien documentée dans la littérature : comme l'a montré Pomerantz 1975, 1984, les tours de parole évaluatifs (ou assessments) interviennent souvent à la fin d'un récit et sont suivis de façon régulière d'une manifestation d'accord (ou de désaccord). C'est seulement après la formulation d'un signe d'accord de la part de $\mathrm{M}$ que $\mathrm{Q}$ reprend la parole en réalisant un tour de parole qui se termine par une question ouverte adressée à l'ensemble des participants et qui oriente ainsi la discussion sur une autre thématique (11. 8-12).

La comparaison entre les deux derniers extraits nous permet d'observer que les constructions clivées peuvent être impliquées dans des séquences de clôture de deux manières : d'une part, le locuteur en cours peut faire intervenir une construction clivée dans la phase de clôture de sa propre contribution verbale (ex. 18); d'autre part, un interlocuteur peut prendre la parole à la fin de l'activité verbale du locuteur précédent et utiliser une construction clivée pour donner une appréciation de ce qui vient d'être dit (ex. 19), en contribuant ainsi à clore un épisode conversationnel. Comme nous l'avons déjà souligné, ce n'est pas uniquement la structure syntaxique de ces énoncés qui oriente les participants vers une activité de clôture. D'autres constructions syntaxiques - comme par exemple la dislocation à gauche - peuvent également être impliquées dans les séquences de clôture (cf. Pekarek Doehler, 2001; De Stefani, 2007). Notons aussi au passage que la notion de "clôture" est utilisée de différentes manières en analyse conversationnelle, où elle est appliqué à la fois à la clôture d'un échange conversationnel, d'épisodes thématiques (ou topicaux) et de séquences conversationnelles (pour un approfondissement v. De Stefani, 2007). Les extraits (18) et (19) présentent en effet des points communs au-delà de la structure syntaxique qui contribuent également à accomplir cette tâche. Nous avons déjà eu l'occasion de relever le caractère 
formulaïque de l'expression c'est pour ça que $Y$; il nous semble que, par sa fréquence, la tournure c'est ça qui compte peut également être considérée comme une expression en voie de figement. Comme l'ont montré Holt \& Drew (2005) et d'autres, les expressions idiomatiques de ce genre et les locutions interviennent régulièrement dans les séquences de clôture topicale. Notons aussi que dans les deux cas la construction clivée implique le pronom démonstratif ça (et non pas, par exemple, des syntagmes nominaux lexicaux) qui, comme l'observe Hancock (2002: 385), est fréquemment observable dans les constructions clivées qui résument les tours de parole précédant son occurrence.

\section{Conclusions}

Les analyses que nous avons menées ont permis d'orienter la recherche sur les constructions clivées vers de nouveaux horizons. L'étude systématique d'un corpus de conversations semi-directives a fait émerger le caractère variable des constructions clivées - qui ne sauraient pas être réduites à des descriptions figées, comme c'est souvent le cas dans les travaux antérieurs. La variation formelle que nous avons observée dans nos données va de pair avec une certaine variation fonctionnelle : nous avons montré que les fonctions focalisante et contrastive communément attribuées aux clivées ne sont pas aisément identifiables à partir du moment où l'on dépasse le niveau de la phrase. Face à la complexité des données, on peut se demander si le couplage forme-fonction - que l'on évoque bien souvent pour identifier les constructions clivées - est un outil adéquat pour la description des structures syntaxiques. D'autant plus que l'analyse de la syntaxe limitée aux aspects structurels et informationnels de la phrase ne permet pas de prendre en compte les fonctions que certaines constructions assurent au-delà du niveau phrastique. En particulier, les tâches que ces constructions accomplissent dans l'interaction se sont révélé être profondément ancrées dans l'environnement séquentiel de leurs occurrences. Comme l'enseigne l'analyse conversationnelle, les unités de la langue sont foncièrement indexicales, c'est-à-dire liées aux contingences interactionnelles locales. Ceci est vrai aussi pour les constructions telles que nous les avons analysées dans cet article : ainsi, nos analyses ont permis de voir que les aspects formels, informationnels et interactionnels sont étroitement liés les uns aux autres. L'examen des extraits interactionnels a révélé que la construction clivée participe à l'organisation et à la gestion des tours de parole, tant au niveau séquentiel qu'au niveau topical. Nous avons montré, en particulier, que les participants mêmes traitent les constructions clivées comme des ressources grammaticales qui permettent d'accomplir des tâches pertinentes pour l'interaction dans laquelle ils sont engagés.

L'objectif de cet article ne consistait pas simplement à montrer qu'une analyse menée du point de vue de la linguistique interactionnelle pouvait permettre de compléter les recherches avancées jusqu'ici dans une perspective fonctionnelle : il s'agissait plutôt de dévoiler la manière dont la structure informationnelle des constructions analysées s'articule avec l'organisation séquentielle du discours. L'adoption d'une perspective émique, qui privilégie le point de vue des participants, et la prise en compte du traitement séquentiel que ceux-ci réservent aux constructions syntaxiques, permet de repenser le système grammatical : la grammaire n'est pas un assortiment de normes, de règles que les locuteurs combinent pour obtenir des phrases acceptables; il s'agit plutôt d'un ensemble de ressources que les membres sociaux peuvent faire intervenir dans leurs interactions pour accomplir des tâches pratiques et socialement pertinentes.

\section{Conventions de transcription}

début de chevauchement

... ... pause brève, moyenne, longue

(1.3) pause en secondes

/ \ intonation montante/ descendante $\backslash$

maison segment accentué 


$\begin{array}{ll}\begin{array}{l}\text { JEUdi } \\ { }^{\circ} \text { demain }\end{array} & \text { haute amplitude de la voix } \\ : & \text { murmuré } \\ ((\text { rire })) & \text { phénomène non transcrit ; remarque du transcripteur } \\ <> & \text { délimitation des phénomènes indiqués entre }((\quad \text { ) }) \\ \text { par- } & \text { troncation } \\ = & \text { enchaînement rapide } \\ \& & \text { continuation du même tour de parole } \\ (\text { il va }) & \text { essai de transcription d'un segment difficilement identifiable } \\ \text { xxx } & \text { segment inaudible } \\ \text { 'h } & \text { aspiration } \\ (\ldots) & \text { partie supprimée de l'énoncé }\end{array}$

\section{Références bibliographiques}

Akmajian, A. (1970). On deriving cleft sentences from pseudo-cleft sentences. Linguistic Inquiry, 1, 149-168.

Auer, P. (2005). Projection in interaction and projection in grammar. Text, 25(1), 7-36.

Ball, C. (1977). Th-clefts. Pennsylvania Review of Linguistics, 2, 57-69.

Berretta, M. (1994). Ordini marcati dei costituenti di frase in italiano. La frase scissa. Vox Romanica, 53, 79-105.

Blanche-Benveniste, C. (2001a). Macro-syntaxe et micro-syntaxe : les dispositifs de la rection verbale. In Andersen, H. L. \& Nølke, H. (éds.), Macro-syntaxe et macro-sémantique, Bern : Peter Lang, 95-118.

Blanche-Benveniste, C. (2001b). Préposition à éclipses. Travaux de linguistique, 42-43, 83-95.

Clech-Darbon, A., Rebuschi, G. \& Rialland, A, (1999). Are there cleft sentences in French? In Rebuschi, G. \& Tuller, L. (éds.), The grammar of focus, Amsterdam/Philadelphia : John Benjamins, 83-118.

Collins, P. (2006). It-clefts and wh-clefts. Prosody and pragmatics. Journal of Pragmatics, 38, 1706-1720.

Croft, W. (2001). Radical construction grammar. Syntactic theory and typological perspective. Oxford: Oxford University Press.

Davidse, K. (2000). A constructional approach to clefts. Linguistics, 38(6), 1101-1131.

Delin, J. (1990). Accounting for cleft constructions in discourse. A multi-layered approach. Edinburgh : Human Communication Research Centre.

Delin, J. \& Oberlander, J. (1995). Syntactic constraints on discourse structure. The case of it-clefts. Linguistics, 33, 465-500.

Deppermann, A. (2006). Construction Grammar - Eine Grammatik für die Interaktion? In Deppermann, A., Fiehler, R. \& Spranz-Fogasy, T. (éds.), Grammatik und Interaktion, Radolfzell : Verlag für Gesprächsforschung, 43-65.

De Stefani, E. (2007). La dislocation à gauche rythmée comme dispositif de clôture séquentielle. In Avanzi, M. \& Horlacher, A.-S. (éds.), Structuration grammaticale et structuration discursive (= Travaux Neuchâtelois de Linguistique, 47).

Frison, L. (1988). Le frasi scisse. In Renzi, L. (éd.), Grande grammatica di consultazione. I. La frase. I sintagmi nominale e preposizionale, Bologna : Il Mulino, 194-225.

Geluykens, R. (1988). Five types of clefting in English discourse. Linguistics, 26, 823-841. 
Geluykens, R. (1991). Discourse functions of it-clefts in English conversation. Communication \& Cognition, 24(3-4), 343-358.

Hancock, V. (2002). L'emploi des constructions en c'est x qui / que en français parlé. Une comparaison entre apprenants de français et locuteurs natifs. Romansk Forum, 16(2), 379-388.

Hedberg, N. A. (1990): The discourse functions of cleft sentences in English. PhD Thesis, University of Minnesota, Minnesota.

Holt, E. \& Drew, P. (2005). Figurative pivots. The use of figurative expressions in pivotal topic transitions. Research on Language and Social Interaction, 38(1), 35-61.

Hopper, P. J. (2004). The Openness of Grammatical Constructions. Chicago Linguistic Society, 40, 239-256.

Hopper, P. J. \& Thompson, S. A. (à paraître). Projectability and clause combining in interaction. In Laury, R. (éd.), Crosslinguistic studies of clause combining : the multifunctionality of conjunctions, Amsterdam/Philadelphia: John Benjamins.

Imo, W. (2007). Der Zwang zur Kategorienbildung. Probleme der Anwendung der Construction Grammar bei der Analyse gesprochener Sprache. Gesprächsforschung - Online Zeitschrift zur verbalen Interaktion, 8, 22-45. [www.gespraechsforschung-ozs.de].

Jespersen, O. (1927). A Modern English Grammar on Historical Principles. Part III, Syntax, Second Volume. Heidelberg : Carl Winters.

Jespersen, O. (1937). Analytic Syntax. New York : Holt, Rinehart and Winston.

Jespersen, O. (1949). A Modern English Grammar on Historical Principles. Part VII, Syntax. Copenhagen : Ejnar Munksgaard.

Katz, S. (2000). Categories of C'est-cleft constructions. Revue canadienne de linguistique, 45(3), 253-273.

Lambrecht, K. (2001). A framework for the analysis of cleft constructions. Linguistics, 39(3), 463-516.

Lambrecht, K. (2004). Un système pour l'analyse de la structure informationnelle des phrases. L'exemple des constructions clivées. In Fernandez-Vest, J. \& Carter-Thomas, S. (éds.), Structure informationnelle et particules énonciatives, Paris : L'Harmattan, 21-61.

Lees, R. B. (1963). Analysis of the 'cleft sentence' in English. Zeitschrift für Phonetik, Sprachwissenschaft und Kommunikationsforschung, 16, 371-388.

Lehmann, C. (à paraître). Information structure and grammaticalization. In López-Couso, M. J. \& Seoane Posse, E. (éds.), New reflections on grammaticalization 3, Amsterdam/Philadelphia : John Benjamins.

Mondada, L. (2001). Pour une linguistique interactionnelle. Marges Linguistiques, 1, 142-162. [http://www.revuetexto.net/Archives/Archives.html].

Muller, C. (2003). Naissance et évolution des constructions clivées en «c'est...que... » : de la focalisation sur l'objet à la focalisation fonctionnelle. In Blumenthal, P. \& Tyvaert, J.-E. (éds.), La cognition dans le temps. Etudes cognitives dans le champ historique des langues et des textes, Tübingen : Niemeyer, 101-120.

Müller, G. (à paraître). La construction pseudo-clivée dans la gestion des séquences conversationnelles. Question de portée. In Apothéloz, D., Grobet, A. \& Pekarek Doehler, S. (éds.), Séquentialité et mouvements dans le discours. Numéro spécial des Cahiers de Praxématique.

Nølke, H. (1983). Quelques réflexions sur la structure sémantique des phrases clivées en français moderne. Modèles linguistiques, 5(1), 117-140.

Nowakowska, A. (2004). La production de la phrase clivée en français : de la syntaxe expressive à la syntaxe dialogique. Modèles Linguistiques, 25, 211-221.

Ochs, E., Schegloff, E. A. \& Thompson, S. A. (éds.) (1996). Interaction and grammar. Cambridge : Cambridge University Press.

Pekarek Doehler, S. (2001). Dislocation à gauche et organisation séquentielle. Marges Linguistiques, 2, 177-194. [http://www.revue-texto.net/Archives/Archives.html]. 
Pierrard, M. (1986). «C'est à mon frère que tu parles » : à propos des « opérations de discours » chez G. Guillaume. Revue Romaine de Linguistique, 31(3), 259-266.

Pomerantz, A. (1975). Second assessments. A study of some features of agreements/disagreements. PhD Thesis, University of California Irvine, Irvine.

Pomerantz, A. (1984). Agreeing and disagreeing with assessments. Some features of preferred/dispreferred turn shapes. In Atkinson, J. M. \& Heritage, J. (éds.), Structures of social action. Studies in conversation analysis, Cambridge : Cambridge University Press, 57-101.

Prince, E. (1978). A comparison of wh-clefts and it-clefts in discourse. Language, 54, 883-906.

Rouget, C. \& Salze, L. (1985). C'est ... qui, c'est ... que : le jeu des quatre familles. Recherches sur le français parlé, 7, 117-139.

Rouquier, M. (2007). Les constructions clivées en ancien français et en moyen français. Romania, 125, 167-212.

Sacks, H. (1992). Lectures on conversation. Oxford : Basil Blackwell.

Schegloff, E. A. (1996). Turn organization. One intersection of grammar and interaction. In Ochs, E., Schegloff, E. A. \& Thompson, S. A. (éds.), Interaction and grammar, Cambridge : Cambridge University Press, 52-133.

Sornicola, R. (1988). It-clefts and wh-clefts. Two awkward sentence types. Journal of Linguistics, 24, 343-379.

Vikner, C. (1973). Quelques réflexions sur les phrases clivées en français moderne. In Actes du $5^{\text {ème }}$ congrès des romanistes scandinaves, Turku : Turun Yliopisto, 221-235.

Wehr, B. (2005). Focusing strategies in Old French and Old Irish. In Skaffari, J., Peikola, M., Carroll, R., Hiltunen, R. \& Warvik, B. (éds.), Opening Windows on Texts and Discourses of the Past, Amsterdam/Philadelphia : John Benjamins, 353-379.

${ }^{1}$ Contrairement à Jespersen, Lambrecht $(2001: 472)$ ne traite pas la partie focale comme un sujet extrapolé, mais bien comme le prédicat de la phrase.

${ }^{2}$ Quant aux aspects sémantiques des constructions clivées, la tradition linguistique (fonctionnelle et générativiste) part du principe qu'il n'y a aucune différence au niveau sémantique entre une phrase clivée et sa réalisation canonique dans le format Sujet-Verbe-Objet. Une critique de cette vision a été exprimée notamment par Geluykens (1988) et Davidse (2000). Nølke (1983) s'exprime sur les aspects sémantiques des constructions clivées en français, en se focalisant surtout sur la nature des présuppositions sur lesquelles se basent les phrases clivées.

${ }^{3}$ Dans cette manière de voir, une phrase Sujet-Verbe-Objet du type Elle a cassé une vitre (inventée) peut être transformée en une pseudo-clivée (Ce qu'elle a cassé c'est une vitrée) et en une clivée (C'est une vitre qu'elle a cassée).

${ }^{4}$ Pour ne donner qu'un exemple en italien, Berretta (1994) explique qu'une phrase Sujet-Verbe-Objet du type (lui) ha comprato il libro 'il a acheté le livre' peut être clivée de deux manières: a) comme è lui che ha comprato il libro, correspondant à la solution française 'c'est lui qui a acheté le livre'; b) comme è stato lui a comprare il libro, littéralement 'ça a été lui à acheter le livre'. Les deux constructions sont interprétées par la plupart des linguistes italiens comme ayant le même effet focalisant.

${ }^{5}$ Notons au passage que la décision de transcrire le pronom elles dans sa forme plurielle - alors qu'au niveau de la prononciation il ne peut pas être distingué de la variante singulière - repose sur l'analyse de ce qui suit dans l'énoncé : il s'agit par conséquent d'une transcription " informée ".

${ }^{6}$ Les trois exemples sont repris de l'article de Muller 2003.

${ }^{7}$ La définition que l'auteur donne des adverbes paradigmatisants est quelque peu vague: «Un adverbe paradigmatisant, c'est pour moi un adverbe dont l'utilisation dans une phrase introduit la présupposition d'un paradigme à partir d'un certain élément de la phrase à laquelle l'adverbe est rattaché » (Nølke, 1983 : 130). L'auteur 
pense surtout à des mots comme même, aussi, surtout, seulement, exactement, par exemple, au moins, (tout) au plus. Il faut souligner toutefois que l'auteur analyse uniquement des occurrences dans lesquelles la position $X$ est occupée d'un adverbe + syntagme nominal et non pas des réalisations dans lesquelles un adverbe seul ou précédé d'une préposition prend la place de l'élément $X$ (comme c'est le cas dans nos exemples (4), (7) et (8)).

${ }^{8}$ La référence de là dans les extraits (10) et (11) reste indéterminée (et n'est probablement pas pertinente pour le bon fonctionnement de l'interaction). On peut supposer que cet élément pointe une partie du discours précédent, mais il est également possible d'y reconnaître une référence temporelle (c'est alors, c'est à ce moment-là...). La récurrence avec laquelle la formule c'est là où/que apparaît dans la langue parlée actuelle nous oriente vers un usage lexicalisé ou idiomatique.

${ }^{9}$ Il est toutefois intéressant de constater que Delin (1990) revendique ce qu'elle appelle une multy-layered approach et qu'elle rejette l'analyse limitée à un aspect unique de la description linguistique : «[...] the choice to use a cleft, and the choice of which cleft to use, depend on a combination of constraints at a variety of levels - syntactic, semantic, and pragmatic - and cannot be summarised at a single level of description » (Delin, 1990 : 17). 